\title{
Relevant redundancy in disjunctive concept learning*
}

\author{
CATHERINE R. KAHRS \\ and \\ ROBERT C. HAYGOOD \\ Arizona State University, Tempe, Ariz. 85281
}

Previous studies of relevant redundancy in multidimensional concept learning have used only conjunctive concepts. In addition, the response schemes have always been fully elaborated, i.e., each combination of levels of the relevant dimensions was represented by a separate category. The purpose of the present study was to extend this work to nonconjunctive concepts and to the more commonly used asymmetrical two-choice response scheme, in which one category is designated as positive and contains all instances meeting the definition of the concept and the other instances are placed in the negative category. The results confirm and extend previous findings in that the addition of redundant relevant dimensions is beneficial both in disjunctive concept learning and with the two-category asymmetrical response scheme. Thus, the beneficial effect of relevant redundancy is shown to be generalizable to other types of conceptual rules and different types of response schemes.

\section{INTRODUCTION}

The effects of relevant redundancy in concept learning have been studied extensively over the last two decades. Notable among the findings have been that increasing relevant redundancy improves performance (Bourne \& Haygood, 1961) but that the form of redundancy, as well as the amount, is important (Bricker, 1955; Haygood \& Bourne, 1964). This work is as yet incomplete for two reasons. First, all of the studies have been done with affirmative or conjunctive concepts, to the exclusion of concepts based on rules other than conjunction, such as disjunctive and relational concepts. Numerous studies of nonconjunctive concepts have been done and, in many cases, the outcomes differ radically from those found with conjunctive rules (e.g., Bower \& King, 1967; Haygood \& Devine, 1967). Thus, it seems useful to demonstrate the existence of a redundancy gain with other than conjunctive concepts.

Second, all multidimensional redundancy studies have presented the $\mathrm{S}$ with a fully elaborated response scheme and have used stimulus dimensions with only two levels. For example, in a two-dimensional problem with color and form as the relevant dimensions, the $S$ would be required to classify the stimuli into four categories: red squares, green squares, red triangles, and green triangles.

*This research was supported in part by a basic research contract (F41609-72-C-0037) with the Air Force Human Resources Laboratory, Flying Training Division. The earlier assistance of Jane L. Mayfield in preparation of stimulus materials and Jean Sandlin in working many of the bugs out of the procedure is gratefully acknowledged. Requests for reprints should be directed to R.C. Haygood, Department of Psychology, Arizona State University, Tempe, Arizona 85281.
In contrast, most recent studies have used an asymmetrical response layout, in which one category is designated positive and contains the stimulus combination that defines the concept (e.g., red squares), while the other category contains all other possible combinations. In previous redundancy studies with the fully elaborated response scheme, each category has contained just one level of an added redundant relevant dimension. In contrast, the asymmetrical response layout requires that two or more levels of a redundant relevant dimension appear in the same category, a feature which might attenuate any redundancy gain. In addition, the use of three-level dimensions, a necessity when nonconjunctive concepts are studied, further complicates the picture. The purpose of the present study was to extend the generality of previous work by comparing the four-choice conjunctive problem (e.g., Bourne \& Haygood, 1961) with two-choice conjunctive and inclusive disjunctive problems, all with three-level dimensions.

\section{METHOD}

The Ss were 96 introductory psychology students who received class credit for participation. All Ss were naive in that they had not previously served in a concept-learning experiment. The Ss were assigned to treatment conditions according to a stratified random procedure, which insured that all groups were filled at the same rate, and each $S$ was run individually.

The experimental design was a 3 by 2 by 2 factorial, with three problem types (two-choice conjunction, two-choice inclusive disjunction, or four-choice conjunction), two levels of redundancy (no redundancy or two redundant relevant dimensions), and two different pairs of relevant attributes (one-triangle or small-yellow). Because this experiment was primarily for verification rather than a parametric study of the variables, conditions involving only a single redundant relevant dimension were omitted. The stimulus patterns were geometric designs which varied in five three-level dimensions: color (red, yellow, or blue), shape (triangle, square, or hexagon), size (large, medium, or small), number (one, two, or three identical figures in a pattern), and background (plain, spotted, or striped). The patterns were mounted on $4 \times 6$ in. cards, which $E$ presented by holding them up directly in front of S. Sorting boxes of the appropriate size and number, properly labeled for the condition being tested, were placed on the table between $\mathrm{E}$ and $\mathrm{S}$. A display listing the dimensions and their levels was available to $S$ throughout the task.

Redundancy was introduced by correlating the levels of two dimensions; for example, if size and number were redundant, small patterns were always single, medium patterns always double, and large patterns always triple. When number and shape were relevant, the two redundant relevant dimensions (size and color) were added in such a way that size was redundant with number and color was redundant with shape; similarly, with size and color relevant, redundancy was added by making shape redundant with size and number redundant with color. In the nonredundant conditions, the extra dimensions were held constant; background was the sole irrelevant dimension for all conditions. In every problem, sufficient extra instances were added to the deck to insure that the proportion of instances in each category was the same.

The task and procedure were essentially the same as those 


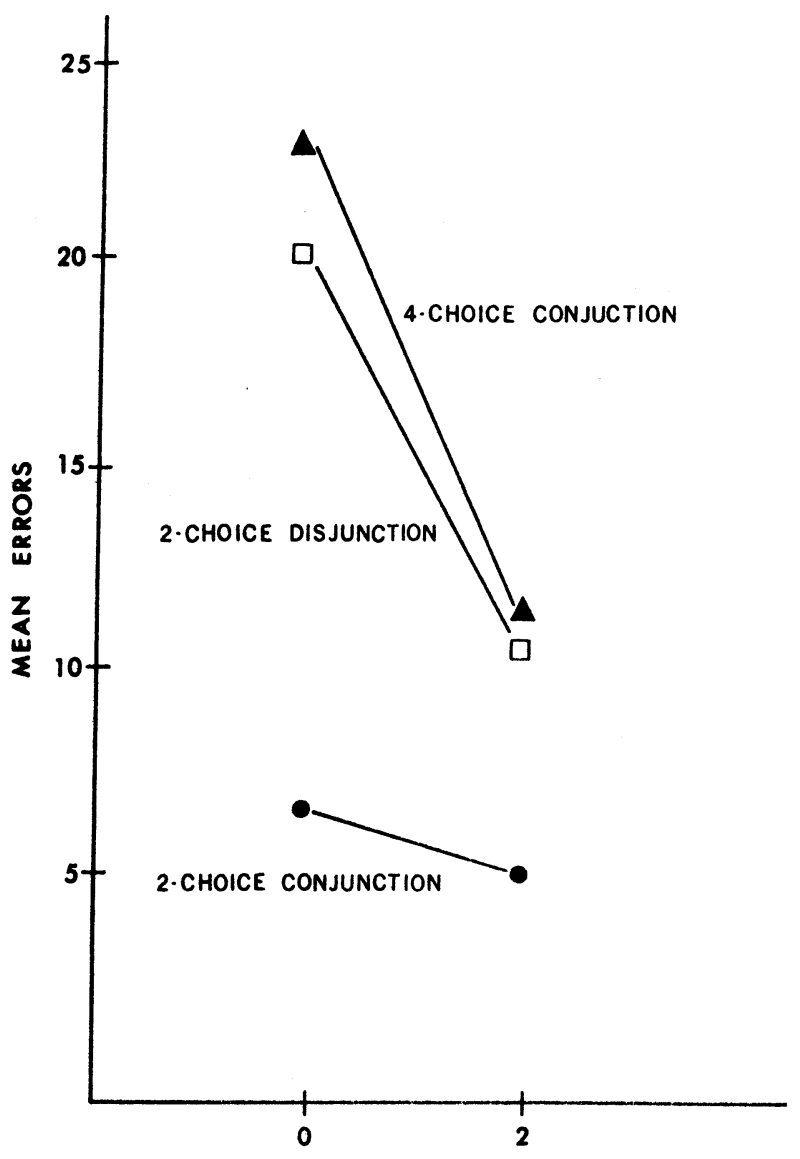

NUMBER OF REDUNDANT DIMENSIONS

Fig. 1. Mean number of errors to solution as a function of number of redundant relevant dimensions for three problem types. Each plotted point represents the data from $16 \mathrm{Ss}$.

described by Haygood \& Bourne (1965). The Ss were required to learn the correct method of sorting stimuli by a process of being shown stimulus patterns, making a classifying response, and receiving corrective feedback. For two-choice problems, the categories were labeled YES and NO to indicate positive and negative instances. In four-choice problems, the four categories were labeled $A, B, C$, and D.

Each $S$ received attribute-identification instructions at the outset which described the stimulus population, the nature of the concept to be learned, the method of responding, the meaning of the feedback, and the criterion of problem solution. The instructions stressed accuracy rather than speed. Following instruction, the stimulus patterns were shown to $S$ one at a time. To each pattern S responded "yes" or "no" (two-choice) or one of the four letters A through D (four-choice). Following each response, $E$ provided feedback by telling the $S$ if he was right or wrong and by placing the card in the correct sorting box. The problem was terminated when $S$ met the criterion of 20 consecutive correct responses or when the time allotted expired. Two Ss were unable to solve in the time available.

\section{RESULTS AND DISCUSSION}

Mean errors to solution as a function of redundancy for the three problem conditions are shown in Fig. 1 . The effect of redundancy is clearly beneficial $[F(1,84)=12.49, p<.01]$. The overall effect of problem type was significant $[F(2,84)=10.03$, $\mathrm{p}<.01]$, but posthoc testing showed no significant difference between the disjunctive and four-choice conjunctive problems. In the overall analysis, the interaction of Redundancy by Problem Type was not significant. However, when the analysis was done for two-choice problems only, the interaction was significant $[F(1,56)=4.58, p<.05]$, with the effect of redundancy greater for inclusive disjunction than for conjunction. None of the other effects was significant.

The results are fully what would be predicted on the basis of previous findings. There is a redundancy gain under all conditions and the gain is greater for the more difficult problems. Among the conjunctive problems, the greater difficulty of the four-choice problem is consistent with previous results (Kepros, 1964). Similarly, among the two-choice problems, the greater difficulty of the inclusive disjunction is comparable to the findings of Haygood \& Bourne (1965). The present results thus provide no surprises, and it appears that previous findings concerning relevant redundancy in affirmative and fully elaborated conjunctive problems can be generalized to nonconjunctive rules and to other response schemes.

\section{REFERENCES}

Bourne, L. E., Jr., \& Haygood, R. C. Effect of redundant relevant information upon the identification of concepts. Journal of Experimental Psychology, 1961, 61, 295-260.

Bower, A. C., \& King, W. L. The effect of number of irrelevant stimulus dimensions, verbalization and sex on learning biconditional classification rules. Psychonomic Science, 1967, 8, 453-454.

Bricker, P. D. The identification of redundant stimulus patterns. Journal of Experimental Psychology, 1955, 49, 73-81.

Haygood, R. C., \& Bourne, L. E., Jr. Forms of relevant stimulus redundancy in concept identification. Journal of Experimental Psychology, 1964, 67, 392-397.

Haygood, R. C., \& Bourne, L. E., Jr. Attribute- and rule-learning aspects of conceptual behavior. Psychological Review, 1965, 72, 175-195.

Haygood, R. C., \& Devine, J. V. Effects of composition of the positive category on concept learning. Journal of Experimental Psychology, 1967, 74, 230-235.

Kepros, P. G. The identification of concepts as a function of stimulus and response complexity. Unpublished doctoral dissertation, University of Utah, 1965.

(Received for publication February 18, 1973.) 